\title{
La prothèse transitoire
}

\section{en prothèse amovible complète ; une optimisation de traitement}

\section{The passing prosthesis in removable prosthesis completes; an optimization of treatment}

\section{Résumé}

\section{MOTS-CLEFS : \\ - Prothèse transitoire, mise en condition tissulaire, mise en condition neuro-muculaire, optimisation de traitement \\ KEYWORDS: \\ - Transitional prosthesis, soft tissue reconditioning, neuromuscular reconditioning, treatment optimizing}

$\operatorname{AOS} n^{\circ} 275-2016$
Avec l'augmentation de l'espérance de vie et la population mondiale vieillissante, le praticien est souvent amené à renouveler les anciennes prothèses complètes de ses patients. Il est alors souvent confronté ò un ensemble de perturbations qui compliquent l'abord des cas ainsi que le pronostic du traitement ; l'attachement affectif des patients ò leur prothèses, l'inflammation douloureuse des surfaces d'appui, quasiment constante, induit par attitude antalgique, un appui occlusal hésitant et une stabilité prothétique improbable. Les tensions musculaires et/ou perturbations articulaires accompagnent souvent une occlusion décentrée à une DVO sous évaluée et un relief cuspidien effacé. La détermination immédiate d'un rapport intermaxillaire stable et répétitif devient alors illusoire. Le recours à la prothèse transitoire permet d'évaluer la possibilité de retour des structures d'appui ò un état de santé tout en optimisant le traitement en terme de confort, de prédictibilité et d'engagement de la part du patient.

L'objectif de ce travail est de passer en revue, à travers un cas clinique, l'attitude clinique ò adopter dans les thérapeutiques de remise en condition, tout en mettant l'accent sur l'intérêt de la prothèse transitoire dans cette approche.

\author{
Abstract
}

With the increasing life expectancy and the aging world population, often, dentists have to renew their patients old complete dentures. Then, they find themselves confronted to plenty of troubles that complicate the approach and the prognosis of the treatment; such as emotional attachment of the patients to their prostheses. Also painful inflammation of the support surfaces, almost constantly, leads to an analgesic attitude, inducing a hesitant occlusal support and an unlikely prosthetic stability. Muscle and / or joint disturbances often go with a non-centered occlusion, an undervaluated vertical dimension and deleted cusp embossing. Trying immediately to fix a steady and repetitive jaw relationship becomes delusive. The use of transitional prosthesis helps evaluating the possibility to find back healthy support structures while optimizing the treatment in terms of comfort, predictability and commitment from the patient.

The objective of this work is to review , through a case report, the clinical attitude to adopt in reconditioning therapy, and highlight the interest of the transitional prosthesis in this approach.
- Omar IRAQUI, Département de prothèse adjointe, CCTD, Rabat, Maroc

S. BERRADA, Département de prothèse adjointe, CCTD, Rabat, Maroc

Nadia MERZOUK, Département de prothèse adjointe, CCTD, Rabat, Maroc

Ahmed ABDEDINE, Département de prothèse adjointe, CCTD, Rabat, Maroc 
$\mathrm{L}$ ors du renouvellement des anciennes prothèses complétes, le praticien est souvent confronté à des perturbations qui compliquent l'abord des cas ; la fibromuqueuse de recouvrement inflammatoire et douloureuse, la DVO éffondrée, les faces occlusales abrasées et les muscles spasmés dans une occlusion de convenance décentrée sont les paramétres d'un tableau clinique couramment rencontré. Ce dernier est le fruit de l'involution progressive des structures d'appuis et de la détérioration des matériux prothètiques (1) (2). L'inflammation de la fibro-muqueuse traumatisée par les anciennes prothèses témoigne de la perte de son osmose. Prendre l'empreinte de tissus ainsi altérés aboutirait à la réalisation d'une prothèse instable. Celle-ci accentuerait l'état tissulaire défaillant, participerait à l'accélération de la résorption de l'os sousjacent et porteraient atteinte à l'équilibre occlusal de la prothèse et par la même à la santé du système neuromusculaire et articulaire du patient. Ces conditions cliniques imposent des thérapeutiques de réadaptation tissulaire et/ou neuro-musculo-articulaire avant toute tentative de réintervention prothétique, sous peine d'aggraver et de fixer les pathologies (3). La prothèse transtoire prend alors toute son importance $(4,5)$ et peut être la prothèse d'usage du patient corrigée ou une nouvelle prothèse réalisée a cet effet. Elle permet de reconditionner les structures neuro-musculo-articulaires et ostéo-fibro-muqueuses tout en remplaçant les dents absentes. Modifiables à volonté, ces maquettes prévisionnelles permettent au praticien ainsi qu'au patient d'évaluer les progrès réalisés en termes de confort, de stabilité et de rétention tout en préservant l'esthétique faciale et la vie sociale du patient. La prothèse transitoire permet ainsi une optimisation du traitement par la prévisibilité des résultats, la réduction du nombre de séances cliniques et du coût du traitement. Le but de ce travail est de développer l'intérêt des prothèses de transition en prothèse amovible complète à travers un protocole clinique spécifique.

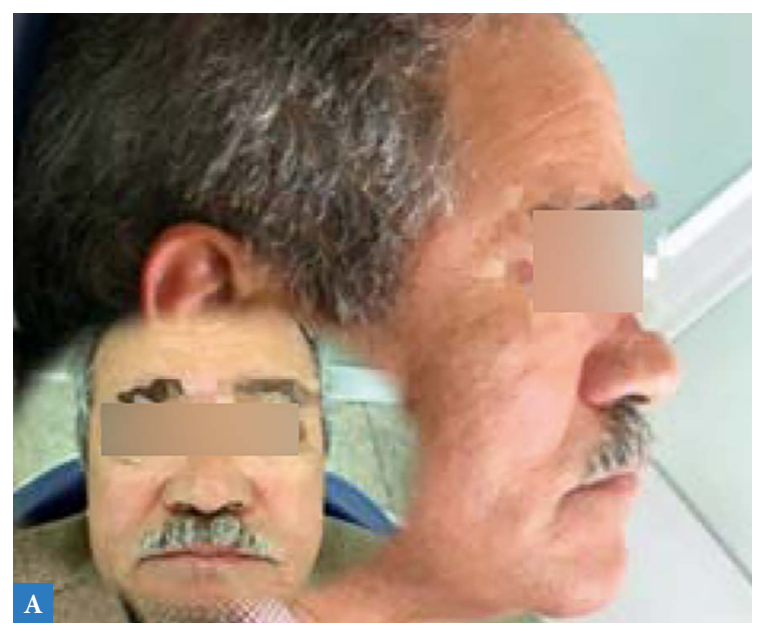

\section{CAS CLINIQUE}

Mr H. M. âgé de 53 ans, en bon état de santé générale consulte pour l'instabilité de sa prothèse complète bimaxillaire. Réalisée 12 ans auparavant, la prothèse n'a bénéficié d'aucune maintenance. La motivation du patient est essentiellement fonctionnelle. Ses habitudes d'hygiène profitent aux prothèses sans porter aucune attention aux crêtes édentées.

\section{L'examen exo buccal}

La DVO imposée par les prothèses était sous évaluée de $6 \mathrm{~mm}$, et accompagnée de spasmes des temporaux et d'une perlèche commissurale (Fig. 1a). L'effondrement de la DVO a été confirmé par l'espace phonétique minimal exagéré à la prononciation $\mathrm{du}$ « $\mathrm{S}$ » (Fig. 1b). Le soutien labial était satisfaisant et les incisives n'étaient pas apparentes à l'état de repos. Au sourire, des fractures des bords libres ont été mises en évidence.

\section{L'examen endobuccal montre}

I Une hygiène défectueuse avec notamment des dépôts de plaque sur les crêtes édentées.

I La fibro-muqueuse recouvrant le versant antérieur du palais est inflammatoire et décollée. Une ulcération est notée sur la zone paratubérositaire gauche (Fig. 2).

$\checkmark$ La crête mandibulaire est régulière et peu résorbée sans contre dépouille marquée. La fibro-muqueuse est inflammatoire et décollée par endroit.

$\checkmark$ La salive est de consistance normale.

I Le rapport inter crête est favorable.

IL'examen fonctionnel montre une mobilité mandibulaire normale sans aucun signe de pathologie articulaire. Des tensions musculaires sont mises en évidence à la palpation des masséters et temporaux.

\section{L'examen des prothèses}

I L'examen des bases : l'état de la résine des bases est généralement acceptable. Au maxillaire, des sur

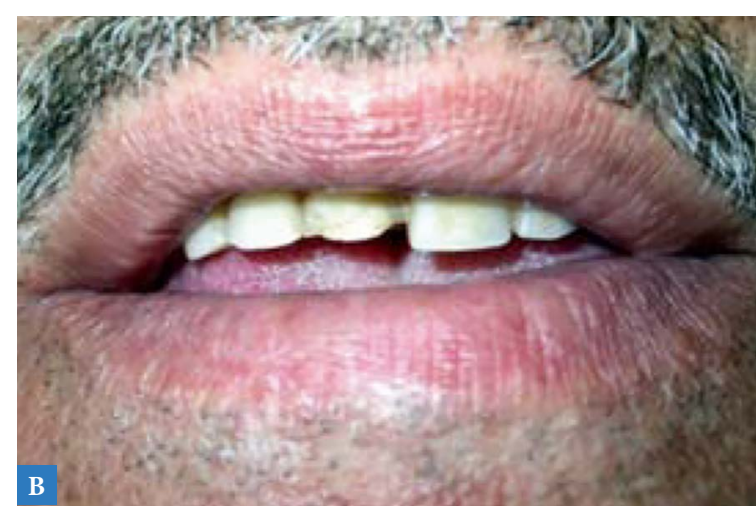

$\triangle$ Fig. 1a : effondrement de l'étage inférieur de face et de profil. Fig. $1 \mathrm{~b}$ : espace phonétique de $6 \mathrm{~mm}$ exagéré à la prononciation du « $S »$. 


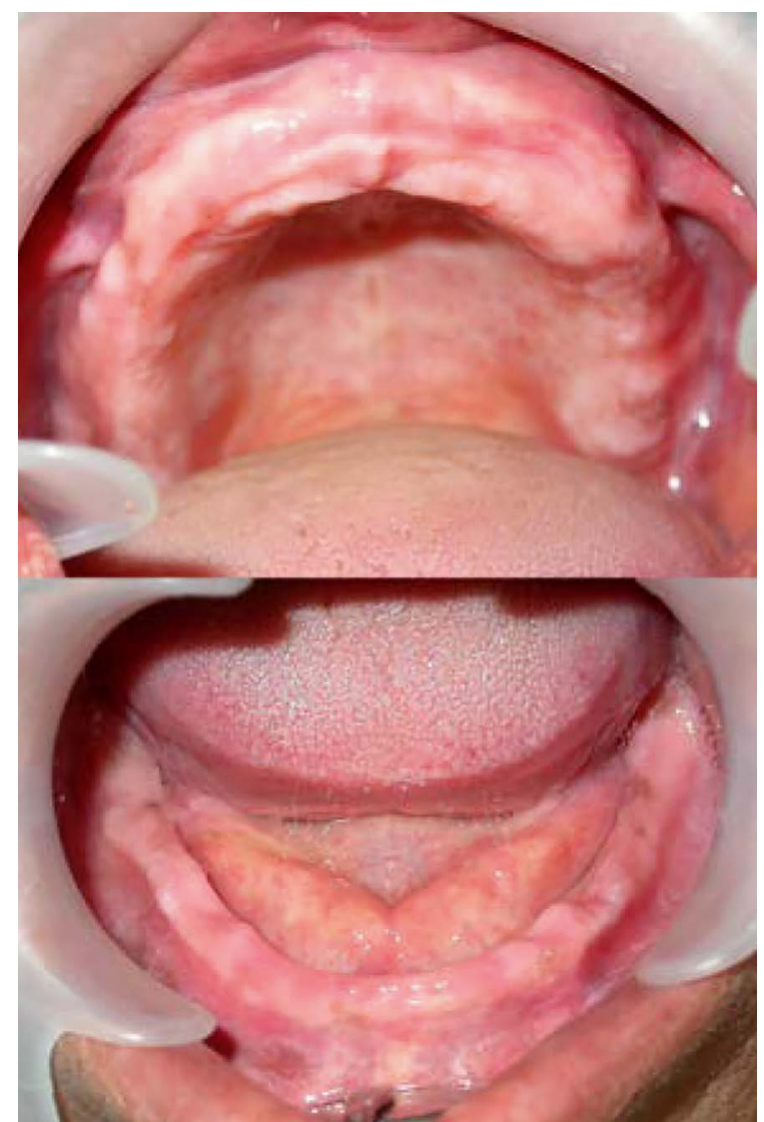

$\triangle$ Fig. 2 : stomatite sous prothétique diffuse généralisée.

extensions des bords antérieurs et latéraux sont confirmées par un matériau révélateur fluide.

I L'examen de l'occlusion montre :

- un plan d'occlusion mal orienté (Fig. 3a);

- une morphologie occlusale abrasée (Fig. 3b) ;

- un décentrage antéro-frontal à la fermeture ;

- des contacts antérieurs en OIM (Fig. 3c);

- absence de contacts équilibrants en latéralité droite. Les conditions prothétiques sont favorables à la transformation des prothèses du patient en prothèse transitoire.

\section{Plan de traitement}

Mise en condition tissulaire

et neuro-musculaire

par le biais des prothèses transitoires

I La mise en condition neuro-musculaire : la position mandibulaire de construction prothétique doit être centrée, stable et pérenne et nécessite un équilibre et une santé musculaire et articulaire. Cet objectif nécessite le traitement préalable des spasmes des temporaux, l'augmentation de la DVO de $6 \mathrm{~mm}$ et le recul centré de la mandibule. Le recours à des plans de morsure plats permet d'effacer progressivement les réflexes de position mandibulaires erronés sans risquer de dépasser les capacités d'adaptation du patient (6). L'efficacité du traitement impose la parfaite fixité des prothèses sur leurs surfaces
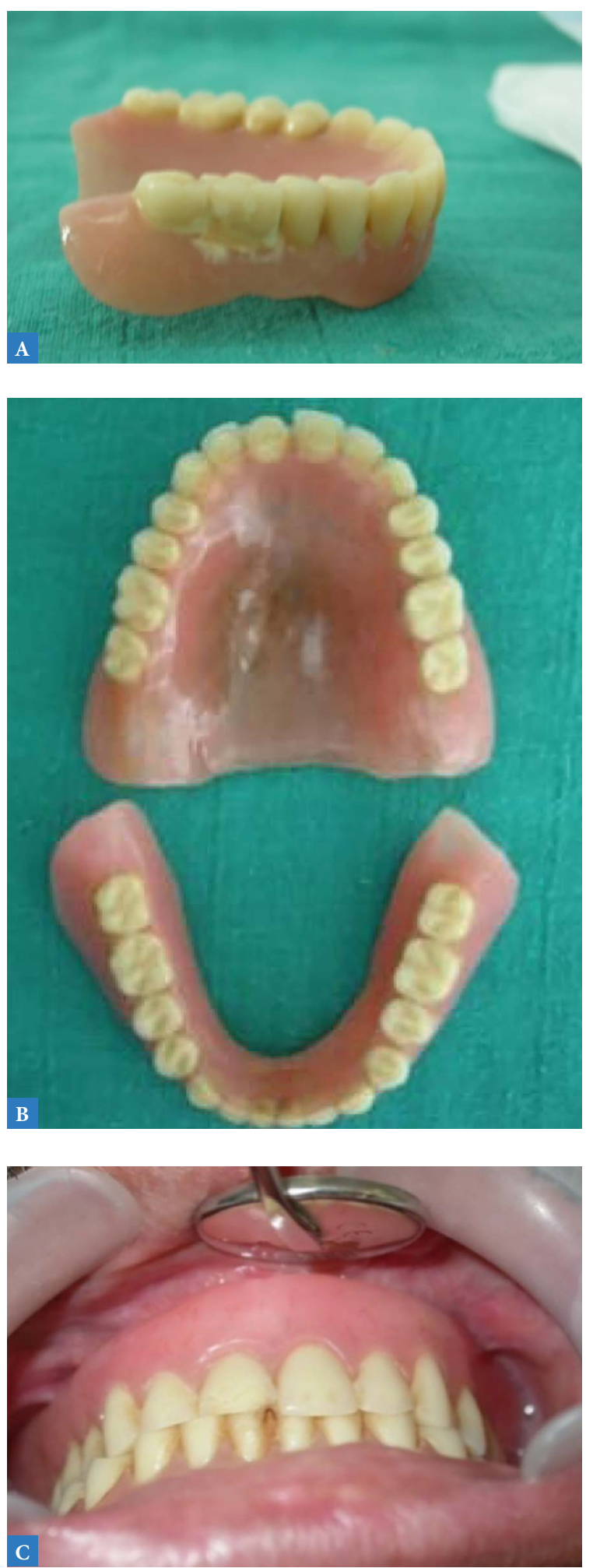

$\triangle$ Fig. 3a : courbe de Spee plate et orientation du plan d'occulation inversée.

Fig. 3b : altération généralisée de la morphologie occlusale. Fig. $3 c$ : contacts antérieurs en OIM.

d'appui. Dans ce sens, la rééducation neuro-musculaire est souvent associée à la mise en condition tissulaire ;

I La mise en condition tissulaire (MET) : associe l'utilisation d'une résine à prise retard véhiculée par des prothèses transitoires à des manœuvres 
d'hygiène adéquates. Outre l'assainissement de la fibromuqueuse et la préservation du capital osseux sous jacent, la MET offre une stabilisation immédiate des prothèses indispensable à l'équilibre occlusal et neuromusculaire du patient.

I Une nouvelle prothèse complète bimaxillaire : intervient après assainissement des structures d'appui directes (fibro-muqueuses et osseuses) et indirectes (neuromusculaires et articulaires). Sa réalisation est spécifique et optimisée, plus courte et plus précise, elle exploite et fixe les résultats obtenus lors des thérapeutiques de reconditionnement tissulaire et neuromusculo-articulaire.

\section{SÉQUENCE OPÉRATOIRE}

\section{Phase préliminaire}

La modification des bases et de l'occlusion des prothèses du patient, ou de leur réplique, est souvent nécessaire et constitue la première phase du traitement. L'innocuité des prothèses transitoires est un préalable indispensable à toute thérapeutique de reconditionnement.

L'interdépendance des facteurs tissulaires, occlusaux et prothétiques dans la fixité prothétique (Fig. 4) fait que l'intervention du praticien cible simultanément l'amélioration de l'ensemble de ces facteurs : l'amélioration des conditions tissulaires du patient passe par l'association d'un contrôle de plaque adéquat à la gestion des contraintes biomécaniques engagées par le port prothétique. Dans ce sens, une stabilité optimale de la base sur sa surface d'appui dans un contexte occlusal équilibré sont des conditions à la

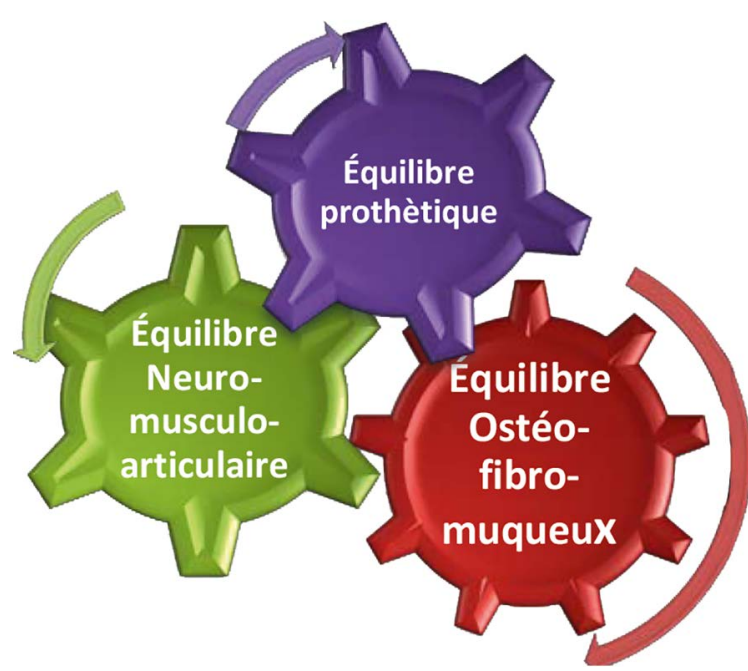

$\triangle$ Fig. 4 : le mauvais état de surface a une influence néfaste sur la stabilité occlusal l'équilibre neuro-musculaire du patient. Parallèlement, une perturbation neuro-musculo-articulaire et/ ou un déséquilibre occlusal perturbent l'équilibre de la prothèse sur sa surface d'appui et donc la santé de cette dernière (Prothèse temporaire en prothèse adjointe. Marcel BEGIN) réussite du traitement. Le traitement des tissus sous prothétiques altérés à l'aide de biomatériaux résineux à prise retard améliore la stabilité des bases prothétiques indispensables à la stabilisation des rapports occlusaux et à l'équilibre neuro-musculaire. Un RIM correct et des contacts occlusaux équilibrés et équilibrants permettent une harmonisation judicieuse des pressions transmises aux surfaces d'appui et favorise la santé tissulaire $(1,4)$.

Pour notre patient nous avons adopté la séquence suivante :

Contrôle des zones de surpression au niveau des bases prothétiques sous pression digitale. En plus de l'altération de l'architecture histologique de la fibromuqueuse de recouvrement et de compromettre la stabilité des tissus supports à moyen terme, elles génèrent des douleurs à la mastication, sources de réflexes d'évitement et de postures antalgiques nuisibles à la thérapeutique de mise en condition tissulaire ou neuro-musculaire. (Fig. 5) (1).

I Stabilisation des bases : l'appui occlusal imposé par les prothèses étant erroné, la mise en condition est réalisée sous pression digitale.

\section{Au maxillaire}

ILes bords de la prothèse maxillaire sont réduits sur $1 \mathrm{~mm}$ de hauteur et en épaisseur et les contre dépouilles éliminées dans l'intrados. L'intrados de la prothèse, ses bords et son extrados sont garnis de Fitt $^{\circledR}$ de Kerr et la prothèse replacée sur sa surface d'appui de façon à ramener le plan d'occlusion à un niveau adéquat plus bas situé (7). Le patient est invité à réaliser les mouvements classiques recommandés en prothèse complète conventionnelle (fig. 6a).

$\checkmark$ La prothèse inférieure est traitée selon le même protocole opératoire; l'intrados prothétique est garni puis la prothèse insérée en bouche, sans contacts occlusaux évitant ainsi tout dérapage relatif aux troubles occlusaux, puis la prothèse est retirée et l'absence de zones de transparence laissant apparaître la base prothétique est contrôlée (Fig. 6b).

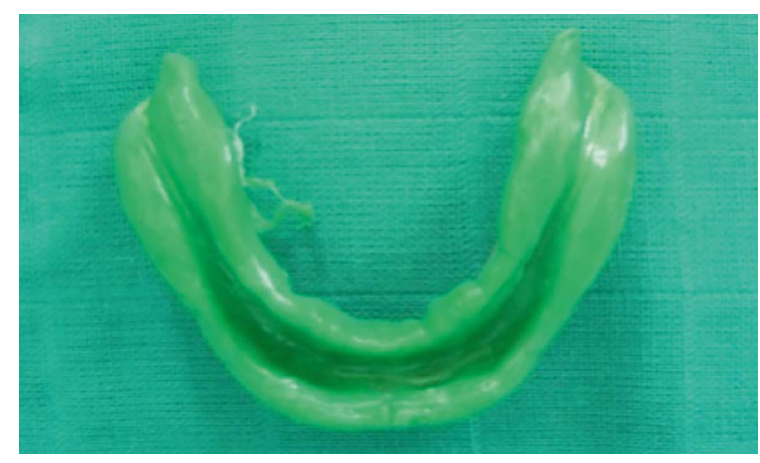

$\triangle$ Fig. 5 : un élastomère fluide sous pression digitale révèle les zones de compression dans l'intrados et les bords prothétiques. 

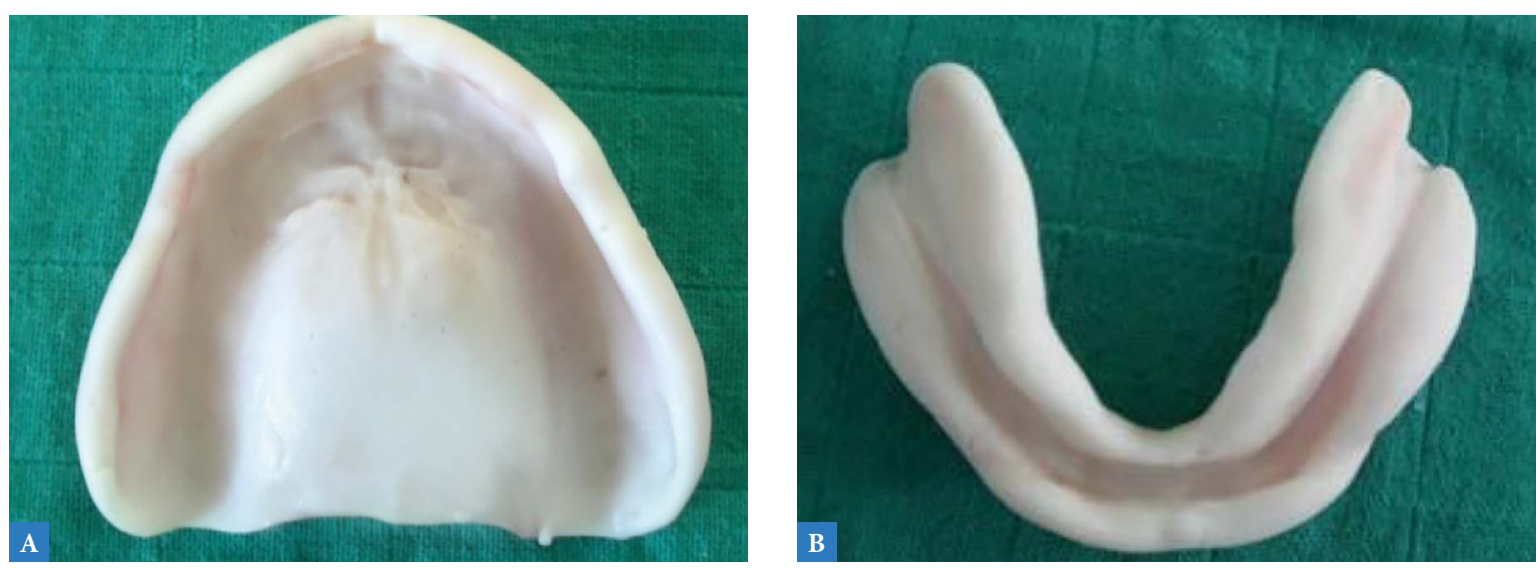

$\triangle$ Fig. 6a : le Fitt ${ }^{\circ}$ de kerr forme une couche épaisse et homogène dans l'intrados prothétique.

Fig. 6b : l'absence de zones de compression dans l'intrados prothétique.

L'objectif est la stabilisation optimale de la base prothétique, l'amortissement des contraintes induites par l'augmentation prévue de la DVO et l'augmentation de la surface de sustentation pour un assainissement efficace des structures d'appui (6)

\section{Mise en condition neuro-musculaire}

La stabilité des bases sur leurs surfaces d'appui acquise, la mise en condition neuro-musculaire est entreprise avec plus de confort et de précision. L'objectif étant d'augmenter la DVO par étapes successives, sans dépasser les capacités adaptatives du patient (espace phonétique minimal préservé). (4) (9)

Le plan d'occlusion maxillaire est réorienté par meulage grâce à la règle de Fox, et la courbe de SPEE est restaurée (Fig. 7). Des plans de morsure sont réalisés : le patient bouche grande ouverte est invité à placer la pointe de la langue vers la gorge (Réflexe Hyo-linguo-mandibulaire) (6), pendant que la fermeture est guidée par le praticien. Grâce aux apports de résine autopolymérisable,

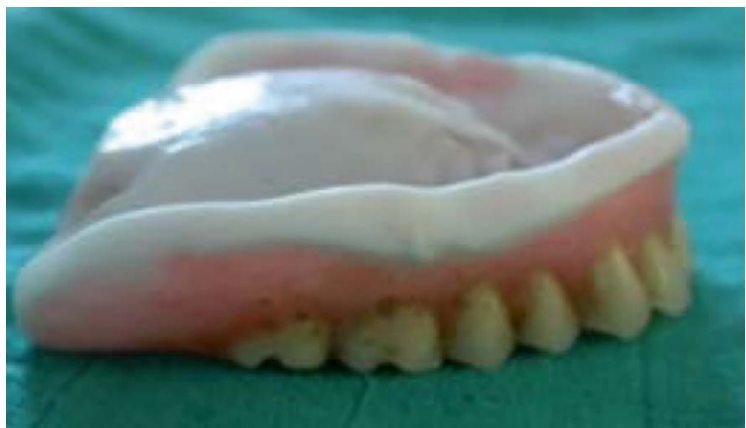

$\triangle$ Fig. 7 : réorientation du plan d'occlusion (règle de FOX), et correction de la courbe de SPEE.

la dimension verticale d'occlusion est augmentée par pallier de $2 \mathrm{~mm}$ en préservant à chaque fois l'espace libre d'innocclusion. Cette augmentation restaure progressivement l'harmonie de l'étage inférieur sans risque de dépassement du pouvoir d'adaptation du patient. Les plans de morsure sont corrigés de manière à obtenir des contacts occlusaux postérieurs simultanés bien répartis de même intensité (Fig. 8) (9).
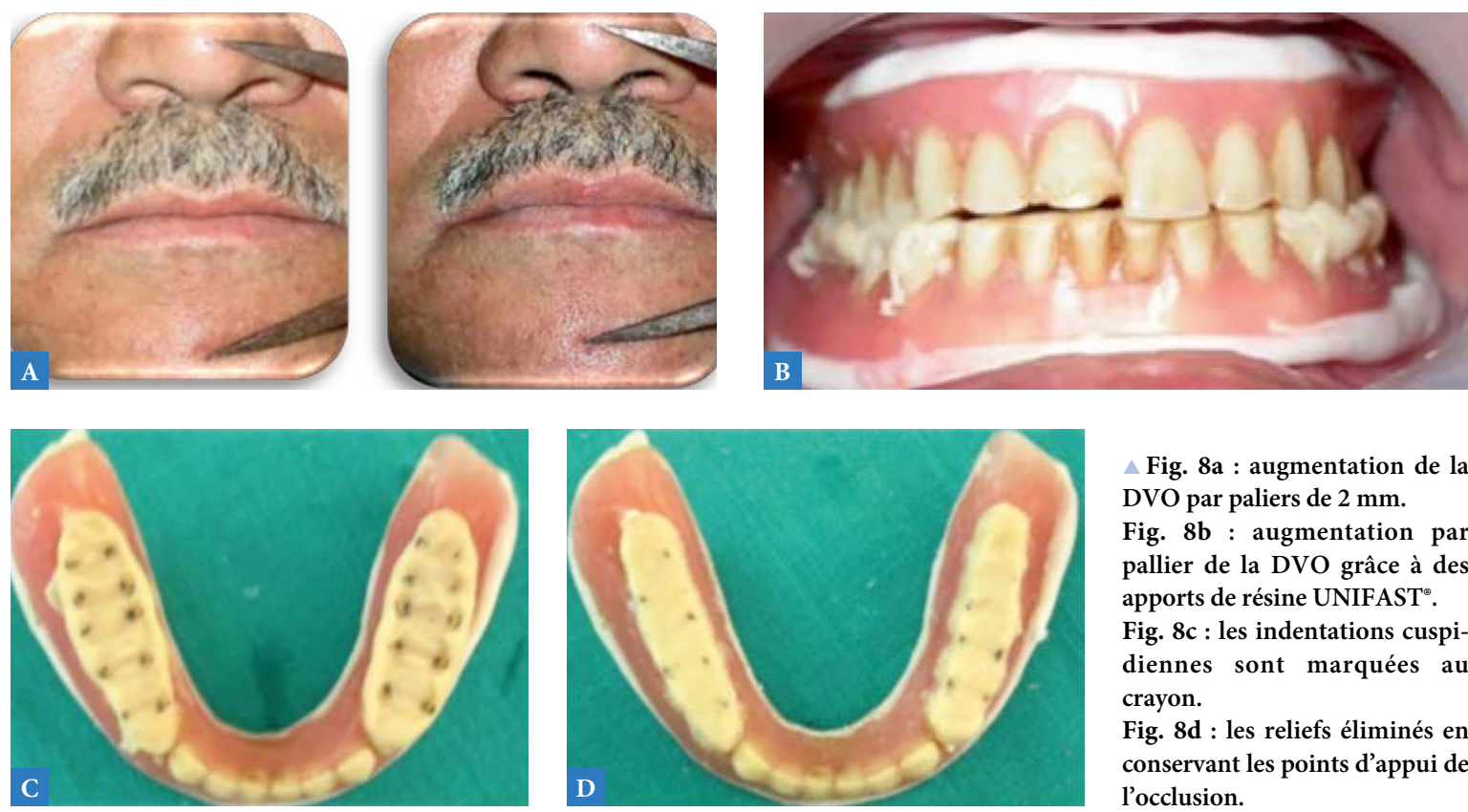

$\triangle$ Fig. 8a : augmentation de la DVO par paliers de $2 \mathrm{~mm}$.

Fig. 8b : augmentation par pallier de la DVO grâce à des apports de résine UNIFAST ${ }^{\circ}$.

Fig. $8 \mathrm{c}$ : les indentations cuspidiennes sont marquées au crayon.

Fig. 8d : les reliefs éliminés en conservant les points d'appui de l'occlusion. 


\section{Suivi hebdomadaire}

Pour la préservation du confort et des capacités d'adaptation tissulaire du patient, l'augmentation de la DVO est envisagée par paliers de $2 \mathrm{~mm}$ jusqu'à obtenir une DVO physiologique. La restitution progressive d'un espace libre d'inocclusion après chaque augmentation signe l'adaptation du patient, et autorise la poursuite du reconditionnement neuro-musculaire (10). Parallèlement, le Fitt ${ }^{\circledR}$ de Kerr est renouvelé chaque semaine jusqu'à disparition de l'inflammation fibro-muqueuse. L'optimisation des effets thérapeutiques passe par l'utilisation d'une consistance fluide améliorant le fluage et la plasticité rémanente du matériau. (Fig. 9) (5) (11).

\section{ÉTAPES DE RÉALISATION DE LA PROTHĖSE DÉFINITIVE}

Au bout de 5 semaines de mise en condition, un état d'équilibre a été atteint. L'assainissement de la fibro-muqueuse, le relâchement des muscles temporaux et la satisfaction du patient en termes de confort et de stabilité prothétique nous ont permis d'aborder les étapes de réalisation de la prothèse définitive.

Les résultats de la thérapeutique de reconditionnement sont transférés et fixés par la prothèse définitive; la résine de reconditionnement tissulaire ayant moulé les structures d'appui dans un état dynamique a joué le rôle d'empreinte anatomo-fonctionnelle ambulatoire. Les plans de morsures ont enregistré le rapport intermaxillaire.

\section{Préalables}

I La précision du RIM établi et le degré de coopération du patient autorisent la réalisation des surfaçages secondaires maxillaire et mandibulaire sous pression occlusale.

I Les prothèses transitoires étant utilisées comme porte empreinte, les éventuelles contre dépouilles de l'intrados prothétique sont éliminées lors de la dernière séance de mise en condition tissulaire. Cette précaution évite ainsi tout risque de fracture du modèle de travail lors de leur désinsertion (12)

\section{$7^{\text {re }}$ séance \\ Clinique}

Le surfaçage du Fitt ${ }^{\circ}$ de Kerr sous pression occlusale réduit le risque de déplacement de l'ensemble surface d'appui-prothèse lors des empreintes maxillaire (12) et assure une répartition idéale des charges occlusales sur les surfaces d'appui (13).

$\checkmark$ Empreinte de la surface d'appui mandibulaire est entreprise en premier lieu et sert d'appui pour l'empreinte sous occlusion du maxillaire. Une faible quantité de Permlastic ${ }^{\circ}$ light garnit la surface du Fitt ${ }^{\circ}$ enduite d'adhésif. La prothèse est insérée en bouche et le patient prié de déglutir et d'exécuter les mouvements préalablement décrits. La dernière phase de polymérisation est assurée sous occlusion (Fig. 10).

I Empreinte secondaire maxillaire est réalisée sous occlusion : le Fitt ${ }^{\circ}$ enduit d'adhésif (Fig. 11a) est recouvert d'une fine couche de Permlastic ${ }^{\circ}$ light puis la prothèse inserée en bouche. Le patient guidé en RC est invité à serrer les dents, puis à exécuter les mouvements préalablement définis. La dernière phase de polymérisation est assurée sous occlusion (Fig. 11b). L'empreinte est retirée et contrôlée (Fig. 11c).

I Enregistrement du rapport inter maxillaire (RIM) et transfert du maxillaire par arc facial : Le RIM défini par les plans de morsure est contrôlé et fixé (Fig. 12); la répétitivité de l'occlusion centrée associée à une DVO physiologique est garante de la persistance des espaces libres physiologiques (ELI, EPM...) et permet de retrouver des fonctions orales équilibrées en alternance avec des périodes de repos (14). La situation spatiale du maxillaire est transférée sur articulateur par arc facial.
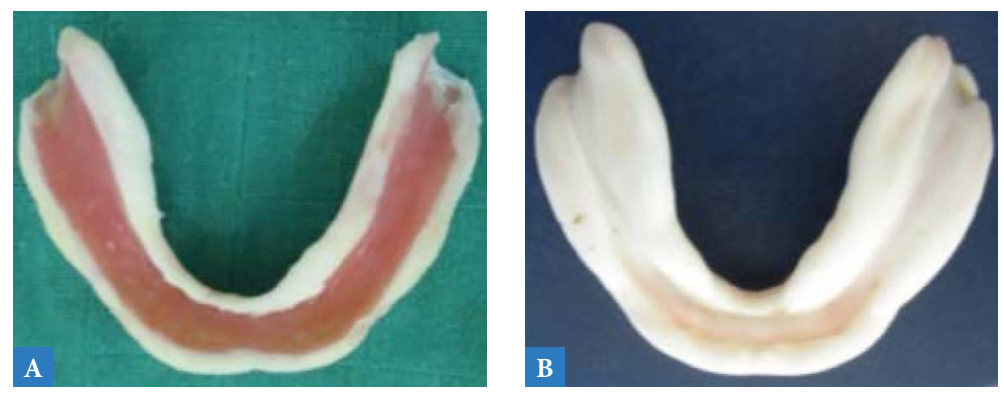

$\triangle$ Fig. 9a : l'évidement central maintient le remarginage ambulatoire pour une stabilité optimale de la prothèse.

Fig. $9 \mathrm{~b}$ : un Fitt ${ }^{\circ}$ de Kerr fluide est préparé en réduisant le rapport poudre liquide pour une plasticité et un fluage améliorés

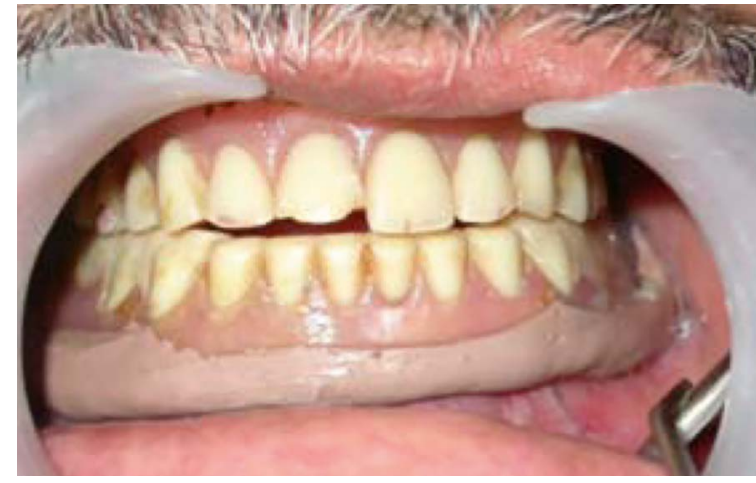

$\triangle$ Fig. 10 : l'empreinte de surfaçage sous occlusion. 

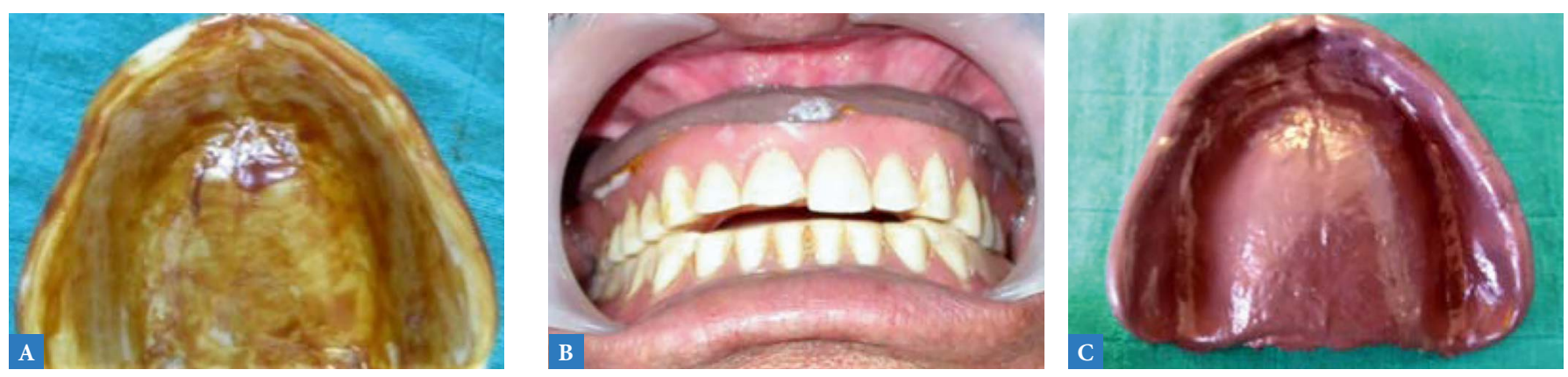

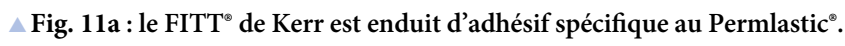

Fig. 11b : l'empreinte anatomo-fonctionnelle sous occlusion.

Fig. 11c : état de surface et précision de l'enregistrement obtenu.

\section{Laboratoire}

I Coulé des empreintes secondaires maxillaire et mandibulaire : les empreintes secondaires sont boxées et coulées en plâtre dur.

I Montage des modèles sur articulateur : sans retirer les prothèses, le montage des modèles sur articulateur débute par le transfert du maxillaire grâce à du plâtre SNOW WHITE ${ }^{\circledR}$. Le modèle mandibulaire est monté par affrontement, tige à 0 (Fig. 13).

I Confection de la maquette d'occlusion : la prothèse supérieure parfaitement stable sur sa surface d'appui est désinsérée et utilisée comme maquette d'occlusion. Les dents prothétiques sont recouvertes d'un bourrelet en plâtre Snow White ${ }^{ø}$ qui est ensuite

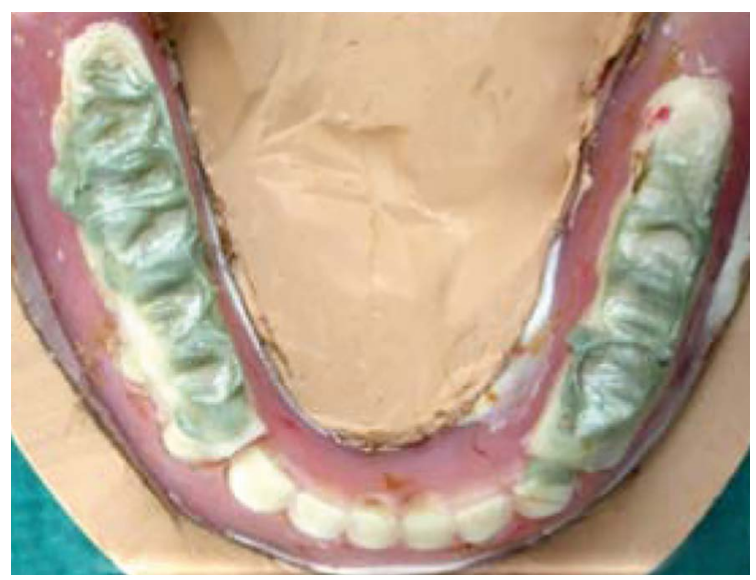

$\triangle$ Fig. 12 : la cire Aluwax ${ }^{\oplus}$ fixe le RIM.

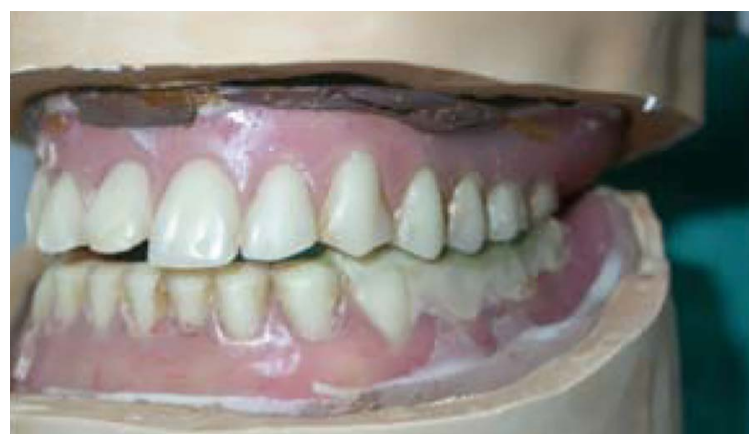

$\triangle$ Fig. 13 : montage des modèles sur articulateur selon le rapport intermaxillaire enregistré. modelé au cutter et au papier abrasif de manière à restaurer la situation idéale du plan d'occlusion.

\section{Deuxième séance \\ Clinique}

Les anciennes prothèses restaurent un soutien labial correct. Ce dernier sera maintenu sur le bourrelet qui laisse apparaître les faces vestibulaires incisives (Fig. 14). L'exposition à l'état de repos ainsi que le parallélisme avec la ligne bi pupillaire et le plan de Camper sont contrôlés à la règle de Fox. Les références esthétiques (point inter incisif, position des faces distales des canines, ligne du sourire) y sont tracées au crayon, et permettent le choix de la dimension des futures dents prothétiques. Ce choix se réfère également aux anciennes prothèses du patient en ce qui concerne la forme des dents ainsi que leur teinte.

\section{Laboratoire}

I La prothèse maxillaire surmontée du bourrelet en plâtre est replacée sur son modèle qui est monté sur articulateur, et permet la réalisation d'une table de montage en silicone, support des références de montage (situation du point inter incisif, position des bords libres) (Fig. 15).

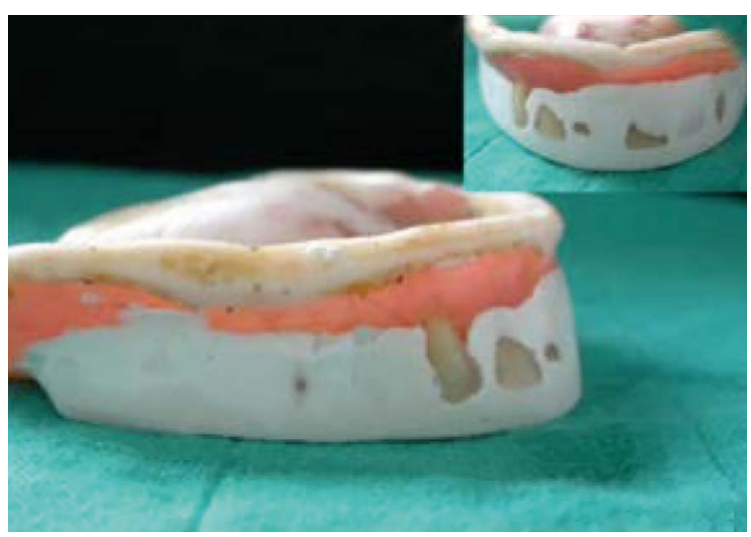

$\triangle$ Fig. 14 : le bourrelet est réglé de manière à obtenir un soutien labial similaire à celui des anciennes prothèses. 


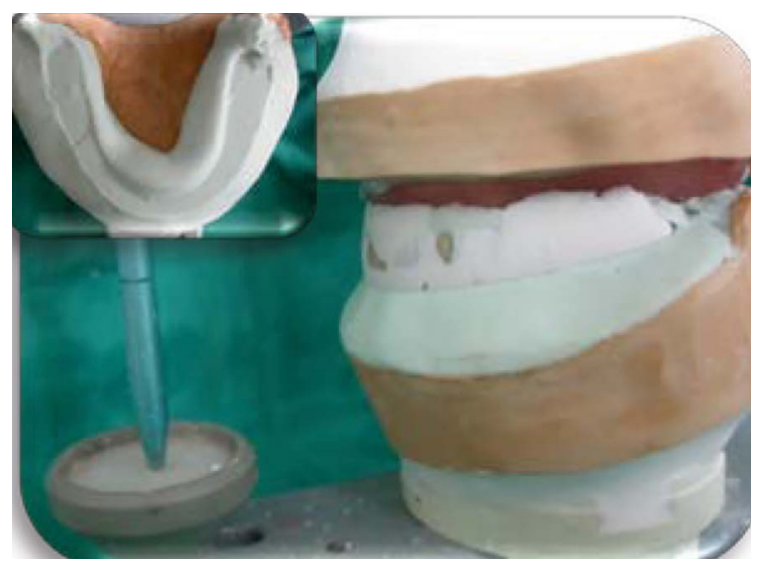

$\triangle$ Fig. 15 : une table de montage en silicone reprend la forme du bourrelet maxillaire.

I Les montages esthétique et fonctionnel sont réalisés en un seul temps sur un articulateur programmé selon des valeurs arbitraires (pente condylienne à $40^{\circ}$ et angle de Bennet à $\left.15^{\circ}\right)(15)$

\section{Troisième séance \\ Clinique}

Les essayages esthétiques et fonctionnels sont abordés dans les mêmes conditions que celles de la prothèse complète bimaxillaire conventionnelle (Fig. 16).

\section{Laboratoire}

Une clé en plâtre est réalisée avant polymérisation des prothèses, et assure un retransfert précis de la prothèse maxillaire polymérisée sur articulateur par la technique de double base engrainée (Fig. 17).

\section{Quatrième séance}

Clinique : contrôle des bases et équilibration immédiate

\Chaque prothèse est soigneusement examinée. Toute zone agressive est adoucie avant de mettre l'appareil en bouche. Au cours de cette séance, les surfaces occlusales des dents non équilibrées ne doivent jamais entrer en contact. Le patient à la recherche de l'intercuspidie obtiendrait une occlusion «de convenance» potentiellement erronée.

$>$ Fig. 18a : des rouleaux de coton interdisent tous contacts entre les tables occlusales non équilibrées. Fig. 18b : enregistrement de l'occlusion par mordu de Tench.

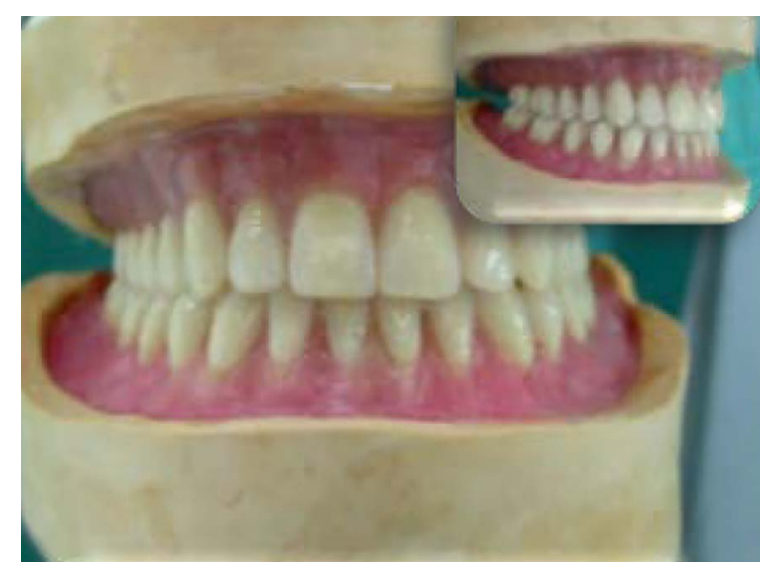

$\triangle$ Fig. 16 : montage esthétique et fonctionnel en OIM.

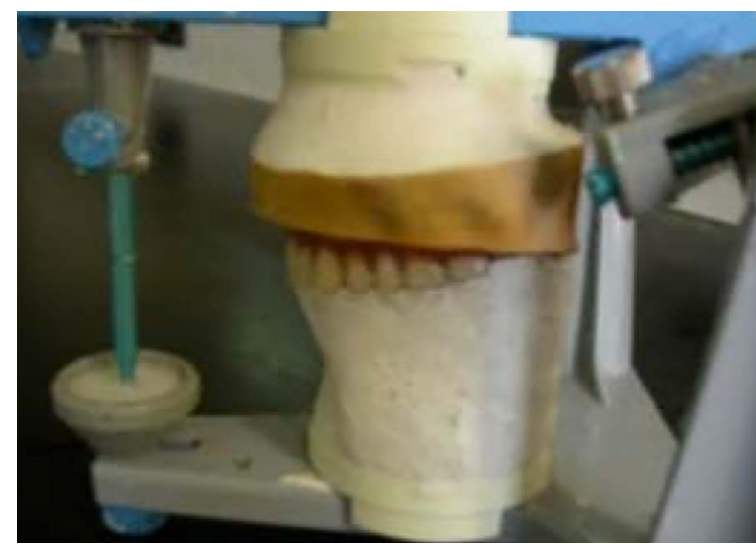

$\triangle$ Fig. 17 : une clé en plâtre est réalisée au stade du montage sur cire.

Après quelques essais, cette position serait adoptée instinctivement ; il serait alors plus difficile de guider la mandibule en relation centrée (16).

I Chaque prothèse est donc essayée séparément. L'insertion correcte de chaque prothèse est contrôlée en faisant serrer le patient sur des rouleaux de coton (Fig. 18a), Cette dernière manœuvre assure une application parfaite des bases sur leurs surfaces d'appui et facilite l'enregistrement de la RC par fatigue musculaire (16).

I Un Mordu de Tench (1) permet un nouvel enregistrement de la relation centrée et le transfert des prothèses sur articulateur pour l'équilibration immédiate de l'occlusion. La faible surélévation de

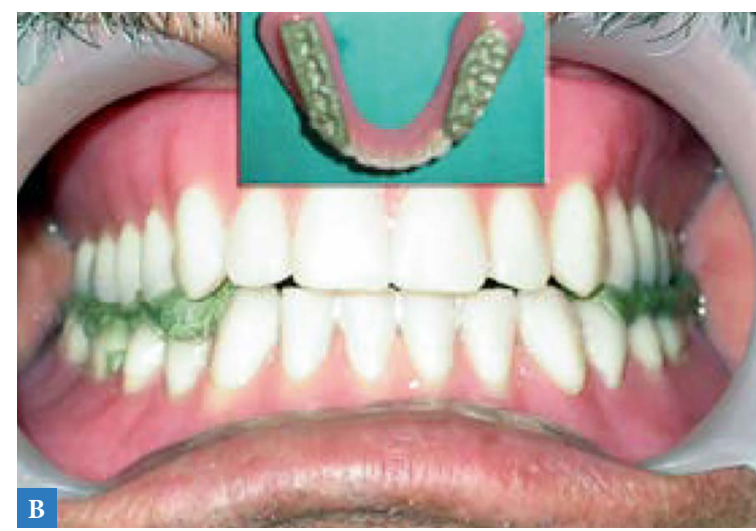


l'occlusion induite autorise l'utilisation d'un axe charnière rapproché selon une précision acceptable (Fig. 18b) (18) (19).

\section{Laboratoire}

L'équilibration des contacts occlusaux est de préférence réalisée par le praticien en présence du patient, selon la séquence classique (16) (17). Elle a pour objectif d'instaurer des contacts occlusaux simultanés postérieurs en relation centrée ainsi que des contacts équilibrants non travaillants en propulsion et latéralités (fig. 19).

\section{Cinquième séance}

Le polissage des faces occlusales est suivie de la pose de la prothèse et des contrôles classiques. Les conseils d'usages sont prodigués au patient, et un rendez-vous est programmé dans les $72 \mathrm{~h}$ (17).

I Séance de contrôle : en appréciant la similitude de confort procuré par ses nouvelles prothèses en comparaison avec les prothèses transitoires, le patient manifeste son entière satisfaction (fig. 20). Les doléances rapportées se sont limitées à une irritation localisée à la zone rétro-mylo-hyoïdienne gauche.

I Discussion : la prothèse amovible complète n'est pas une prothèse définitive, elle doit être assimilée à une prothèse d'usage et être accompagnée d'une maintenance régulière. En effet, le suivi prothétique permet de maintenir l'équilibre du système manducateur dans une dynamique en perpétuelle évolution faisant face à l'interaction des phénomènes d'involution osseuse et des perturbations occlusales et prothétiques relatives aux vieillissement des structures et des matériaux (1) (2). Tout manquement à cette règle induit l'apparition d'altérations fonctionnelles et structurelles plus ou moins réversibles dites adaptations acquises. Leurs objectifs est le maintien de la fonctionnalité des prothèses malheureusement au dépens de la

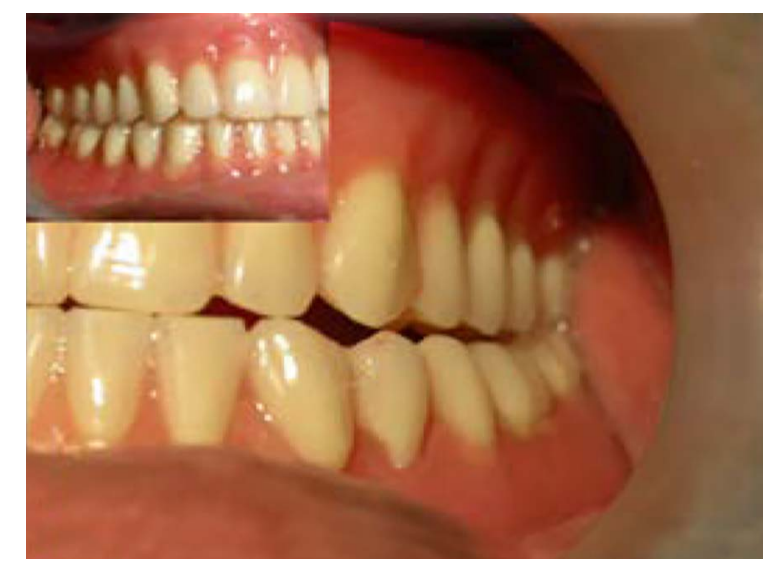

$\triangle$ Fig. 19 : contacts équilibrants en propulsion.

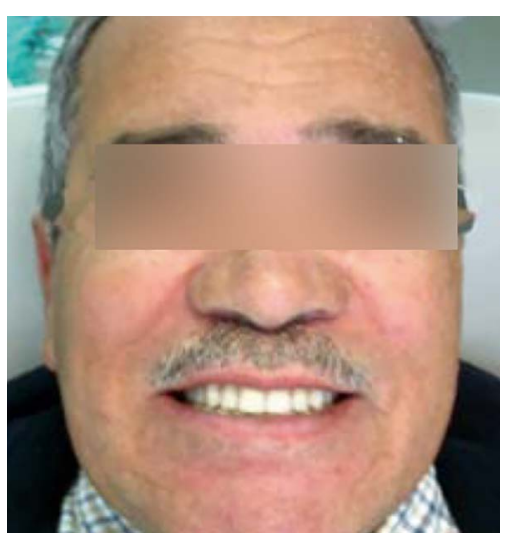

$\triangle$ Fig. 20 : sourire de satisfaction du patient.

santé des différentes structures supports. Dans ce cas, la prothèse transitoire s'avère souvent incontournable; elle reconditionne les structures d'appui directes et indirectes et permet le retour du système masticateur à un état de santé. Les prothèses définitives exploitent l'état d'équilibre ainsi atteint, et pérennisent les résultats obtenus (3). L'intérêt de la prothèse transitoire se manifeste également par l'optimisation du traitement prothétique de par la prévisibilité des résultats et la réduction du nombre de séances cliniques et du coût du traitement (Tab. 1).

\begin{tabular}{|l|}
\hline \multicolumn{2}{|c|}{ Protocole classique } \\
Thérapeutique de mise en condition tissulaire \\
\hline Empreinte primaire \\
\hline Réalisation du PEI \\
\hline Empreinte secondaire \\
\hline Maquette d'occlusion \\
\hline \multicolumn{2}{|c|}{ Rapport intermaxillaire } \\
\hline Essayages esthétique et fonctionnel \\
\hline Pose et équilibration \\
\hline
\end{tabular}

$<$ Tableau 1: Réduction du nombre des séances cliniques et de laboratoire par l'utilisation des prothèses transitoires 


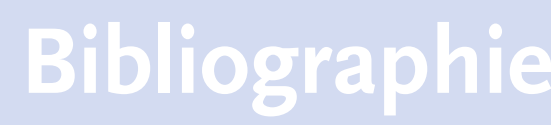

[1] Lejoyeux J. La réfection des bases en prothèse complète. $C d P$ édit, Paris 1995.

[2] Lejoyeux J. Prothèse complète. Tome 2 : diagnostic, traitement. Maloine édit, Paris, 1976.

[3] Begin M, Mollot P. Prothèses temporaires en prothèse adjointe. Réal. Clin 1994; 5: 75-90

[4] Hamel L, Giumelli B, Amouriq Y, Le Bars P. Réhabilitation occlusale par prothèse amovible complète. Encycl Méd Chir, Odontologie, 23-325-M-10, 2000.

[5] Tosello A, Chevaux J-M. Réfection des bases prothétiques. Encycl. Méd Chir, Odontologie, 23-325-H-10, 2001.

[6] Lejoyeux J, Lejoyeux R. Mise en condition en prothèse amovible. 2éd. Masson édit, Paris, 1993
[7] Benfdil N, Merzouk E, Rahmani, A, Benamar, A. Abdedine F. Intérêt de la mise en condition tissulaire dans la remise en état des muqueuses buccales. Actual Odonto-Stomatol 2003; 222: 12-132.

[8] Rignon-Bret C. Porte-empreintes individuels en prothèse adjointe complète. $\mathrm{Cah}$. Proth 1999; 107: 17-28.

[9] Jaudoin P, Millet C, Mifsud S. Empreintes en prothèse complète. Encycl. Méd Chir, Odontologie 23-325-C-10, 2006.

[10] Millet C, Jeannin C, Jaudoin P, Dimensions verticales en prothèse complète. Encycl. Méd Chir, Odontologie 23-325-C-10, 2005.

[11] Ciancaglini R, Sgarzini, Sgarzini L. Rôle de la prothèse transitoire dans la réhabilitation crânio-mandibulaire. Réal Clin 1994; 5: 63-73.

[12] Nanfi C, Tosello A, Preckel E, Pesci-Bardon C. Les résines à prise retardée : utilisation rationnelle en fonction de leurs propriétés physico-chimiques. Cah Proth 2000; 111: 43-52.
[13] Rignon-Bret C, La réadaptation des prothèses amovibles totales. Cah Proth 2000; 110: 35-45.

[14] Hue O, Berteretche MV. Prothèse complète. Réalité clinique. Solutions thérapeutiques. Quintessence International édit, Paris, 2004.

[15] Begin M, Hutin I, Le rapport intermaxillaire en prothèse adjointe complète. 1997; 8: 389-407.

[16] Orthlieb JD, Laplanche O, Preckel EB. La fonction occlusale et ses dysfonctionnements. Real Clin 1996; 72: 131-146.

[17] Berteretche MV. L'équilibration en prothèse adjointe totale. Actual. Odonto-Stomatol 1996; 196: 559-79.

[18] Berteretche MV, Hue O. Insertion et équilibration occlusale. Encycl. Méd Chir, Odontologie 23-325-C-10, 2005.

[19] Dupas PH. Comprendre l'articulateur au cabinet dentaire et au laboratoire. $C d P$ édit, Rueil Malmaison, 2001.

[20] Orthlieb J-D, Laborde G, Bezzina S, Gros $P$. Rôle de l'articulateur dans la transmission des données du cabinet au laboratoire. Réal Clin 2002; 13: 109-123. 